\title{
La actividad investigadora de la universidad española en la primera década del siglo XXI: la importancia del tamaño de la universidad
}

\author{
Teodoro Luque-Martínez* \\ *Dpto. Comercialización e Investigación de Mercados, Facultad de Ciencias Económicas y Empresariales, Universidad de Granada \\ Correo-e: tluque@ugr.es
}

Recibido: 26-11-2012; 2a version: 16-03-2013; Aceptado: 15-04-2013.

Cómo citar este artículo/Citation: Luque-Martínez, T. (2013). La actividad investigadora de la universidad española en la primera década del siglo XXI: la importancia del tamaño de la universidad. Revista Española de Documentación Científica, 36(4):e026. doi: http://dx.doi.org/10.3989/redc.2013.4.1046

Resumen: Este trabajo analiza la actividad investigadora de las universidades públicas presenciales españolas durante la primera década del siglo XXI, a partir de los datos del Observatorio IUNE que recoge datos de I+D+i de fuentes oficiales. Se analiza si la antigüedad, el tamaño (medido por número de profesores o por el número de estudiantes) y la ratio estudiantes por profesor de las universidades genera diferencias significativas en los indicadores de la actividad investigadora, diferenciando por reconocimiento (número de sexenios), actividad científica (producción, colaboración, impacto, visibilidad), innovación, competitividad y capacidad formativa.

Se comprueba la importancia del tamaño de la universidad, por número de profesores, que presenta mejores indicadores para las universidades más grandes y que anula en gran medida el efecto de la antigüedad de la universidad o de la ratio estudiantes por profesor.

Palabras clave: Actividad investigadora universitaria; tamaño de la universidad; antigüedad de la universidad; ratio estudiantes-profesor; I+D+i en la universidad.

Research activity of Spanish universities in the first decade of the 21st century: the importance of the size of the university

Abstract: This paper analyses the research activity of Spanish public universities during the first decade of the 21st century, based on data of R \& D collected by the IUNE Observatory from official sources. We analyse whether the age, size (measured by number of faculty members or number of students) and student-teacher ratio of universities generate significant differences between research activity indicators, distinguishing between recognition (number of 6-year research periods), scientific activity (production, collaboration, impact, visibility), innovation, competitiveness, and training capacity. The importance of the size of the university (number of teachers) is confirmed, as the largest universities had better indicators, and the effect of both the age of the university and the student-teacher ratio was nullified.

Keywords: Research activity; university size; university age; student-teacher ratio; university R\&D.

Copyright: (c) 2013 CSIC. Este es un artículo de acceso abierto distribuido bajo los términos de la licencia Creative Commons Attribution-Non Commercial (by-nc) Spain 3.0. 


\section{INTRODUCCIÓN}

La universidad forma parte del sistema de educación superior junto con una creciente diversidad de instituciones entre las que se teje una red de colaboraciones y rivalidades al mismo tiempo. Como indica Rothblat (2012) cada vez más la universidad es "multidiversidad", por un lado la educación-formación, por otro el conocimiento, la investigación, el descubrimiento, la respuesta a problemas o el compromiso con el desarrollo. El significado de "universidad" no puede ser uniforme o estable de manera que preguntarse por el futuro de la universidad puede ser redundante (Barnett, 2012). Interesa conocer el papel de la universidad en este contexto pero la medida del desempeño, sea de la universidad o del sistema de educación superior, es un asunto extremadamente complejo que ha propiciado la aparición de numerosas iniciativas y ranking.

En la última década han proliferado los rankings de universidades que miden diferentes dimensiones de la actividad universitaria y eso tanto en ámbito de países concretos (por ejemplo EEUU, Francia o España) como en ámbito internacional, es el caso del Academic Ranking of World Universities (ARWU) de Shanghai, el de Times Higher Education, el Webometrics Ranking of World Universities una iniciativa del Cybermetrics Lab del Consejo Superior de Investigaciones Científicas (CSIC) o el Performance Ranking of Scientific Papers for World Universities (PRSPWU) elaborado por Higher Education Evaluation and Accreditation Council of Taiwan (HEEACT), que recientemente ha pasado a denominarse National Taiwan University Ranking o NTU Ranking, sin olvidar la iniciativa del Muti-dimensional Global University Ranking, U-Multirank.

El ranking que ha alcanzado mayor notoriedad es el ARWU de Shanghai, con alta correlación con el PRSPWU (Chen y Liao, 2012), de manera que sirve de referente para evaluar las actuaciones de las universidades y comparar sistemas de educación superior (Docampo, 2012; Docampo, 2011) o la iniciativa de campus de excelencia internacional en España (Docampo y otros, 2012).

También han surgido otras iniciativas de carácter específico como el IPCI (Intellectual Property Citation Index del Science and Technology Policy Research and Information Center (STPI, Taiwan), el Green Metric World University Ranking de la Universidad de Indonesia sobre la sostenibilidad en la universidad o la iniciativa del International Herald Tribune sobre la empleabilidad en las universidades con el Informe Global de Empleabilidad 2012 (Global Employability Survey 2012).

No obstante, estas iniciativas hacia temáticas específicas, la actividad investigadora tiene, y debe tener, un peso especial en la elaboración de los rankings internacionales. La medida de la actividad investigadora es objetivo de investigaciones para medir su productividad o impacto (Jiménez-Con- treras y otros, 2011), entre otras de sus manifestaciones. La actividad investigadora tiene un carácter multidimensional puesto que hay que contemplar el volumen de producción pero también los indicadores de calidad o de transformación de esa producción en desarrollo e innovación. Precisamente, lo que se denomina como la paradoja europea se constata por la diferencia que hay en Europa, comparada con otras zonas (en particular con EEUU), entre la excelencia científica medida por el número de publicaciones, por un lado, y la capacidad de innovación y de emprendimiento, en definitiva de la aplicación de esa potencialidad científica, por otro (Herranz y Ruiz-Castillo, 2012).

Partiendo de la consideración en sentido amplio de actividad investigadora, como la sostenida en el Observatorio IUNE, este trabajo tiene como objetivo fundamental analizar los efectos de la antigüedad y del tamaño de las universidades españolas en su actividad investigadora durante la primera década del siglo XXI. En concreto, se muestra la incidencia, tanto en magnitudes absolutas como relativas atendiendo al número de profesores, que sobre:

- la producción, la colaboración, el impacto y la visibilidad de la actividad científica,

- la innovación,

- la competitividad,

- la capacidad formativa

tiene:

- la antigüedad de la universidad

- el tamaño de la universidad, medido por el número de profesores y número de estudiantes matriculados y a tiempo completo,

- la ratio estudiantes por profesor.

En definitiva, se trata de descubrir si las universidades más antiguas son más productivas, innovadoras, competitivas o con mayor capacidad formativa que las universidades más recientes. Así como de saber si las universidades más grandes, en comparación con las más pequeñas, son más productivas, innovadoras, competitivas o con mayor capacidad formativa.

\section{LA MEDIDA DE LA ACTIVIDAD INVESTIGADORA}

Efectivamente, el primer desafío es el problema de medida. Cómo medir la actividad investigadora y homologar el proceso de medida es algo fundamental para hacer estudios comparativos entre entidades, entre territorios y a través del tiempo para conocer la evolución de las universidades y del sistema de educación superior.

Para comenzar es necesario un buen proceso de recogida de datos y en eso se ha progresado bastante en los últimos años con medios, procesos y 
estructuras en la universidades para avanzar con unidades de bibliometría (Torres-Salinas y otros, 2012), incluso mediante la creación de bases de datos institucionales producidas por la propia universidad (Filippo y otros, 2011).

El interés sobre el análisis de la actividad investigadora de las universidades es objeto de continuas mejoras metodológicas para conseguir medidas que reflejen mejor el estado y la evolución de dicha actividad por universidad y para las distintas dimensiones, como la representación de las relaciones entre las publicaciones de las universidades (García y otros, 2012).

La calidad del registro y el conocimiento de la evolución de la actividad investigadora favorecen una mejor toma de decisiones en la gestión de las universidades en dos grandes ámbitos. Por un lado, a nivel interno para orientar la producción científica (Li y otros, 2012), la gestión de la política científica y de $I+D+i$ de las propias universidades y su estrategia, tanto en ámbito nacional como internacional (Sun y Liu, 2012; Lin y Roberts, 2012). Por otro lado, y en relación con el entorno, para conocer las consecuencias de dicha actividad investigadora en el entorno, en el desarrollo social y económico. De esta manera se puede conocer la implicación en el tejido industrial o la colaboración público-privada en las actividades de conocimiento, de innovación y de desarrollo (Abramo y otros, 2012). En definitiva, las relacionadas con el modelo de la triple hélice (Etzkowitz y Leydesdorff, 1998) y constatar si se revalida la evidencia de una relación positiva entre investigación y actividades de $\mathrm{I}+\mathrm{D}+\mathrm{i}$ y el desarrollo territorial (Jaffe, 1989; Acs y otros, 2002; OCDE, 2007, 2011).
En España una de las medidas más completas de la actividad investigadora de las universidades es la recogida, desde el año 2012, por el Observatorio IUNE coordinado por el Laboratorio de Estudios Métricos de la Información (LEMI) (Sanz-Casado (2012). Se trata de una herramienta de gran valor para nuestro objetivo y que contiene los datos de la primera década del siglo XXI, desde el año 2002 al 2010 agrupados en las dimensiones de la tabla I, y procedente de fuentes oficiales, por lo que son los más fiables y homogéneos entre los disponibles.

Estas dimensiones se descomponen en una serie de indicadores recogidos en la tabla cuyas etiquetas comienzan con la inicial de la dimensión. Es decir, P_ para PROFESORADO; R_ para Reconocimiento; AC_ para ACTIVIDAD CIENTIFICA; IN_ para INNOVACIÓN; COM_para COMPETITIVIDAD; y CF_para CAPACIDAD FORMATIVA, referidos a valores totales o promedios (señalados por TOT o PROM) o al número de profesores (se indican por 100P).

Además hay que señalar que la dimensión ACTIVIDAD CIENTÍFICA se divide en:

- "Producción/productividad" que agrupa los indicadores AC_PUBL_PROME y AC_PUBXPROF_PROMD.

- "Colaboración" contiene a AC_COAUTO_ PROM; AC_COLABINTER_PROM.

- "Impacto" formado por AC_CITAS_TOT; AC CITASPROF_PROM; AC_DOCNC_PROM.

- "Visibilidad" comprende: AC_1C_S_TOTAL; AC_1C_PROM; AC_TOP3_TOT, AC_ TOP3 \%TOT.

Tabla I. Dimensiones de la actividad investigadora y fuente de información

\begin{tabular}{ll}
\hline & \\
\hline P_: Profesorado & INE (Instituto Nacional de Estadística). \\
\hline R_: Reconocimiento & $\begin{array}{l}\text { CNEAI (Comisión Nacional de Evaluación de la Actividad Investigadora) / Ministerio de } \\
\text { Ciencia e Innovación. }\end{array}$ \\
\hline AC_: Actividad científica & $\begin{array}{l}\text { Plataforma Web of Science (Science Citation Index, Social Science Citation Index, y Arts } \\
\text { \& Humanities Citation Index). }\end{array}$ \\
\hline IN_: Innovación & $\begin{array}{l}\text { Red OTRI (Encuesta anual a las universidades) / } \\
\text { INVENES (creada por la Oficina Española de Patentes y Marcas). }\end{array}$ \\
\hline COM_: Competitividad & $\begin{array}{l}\text { CDTI (Centro para el Desarrollo Técnico Industrial) / MICINN (Ministerio de Ciencia e } \\
\text { Innovación). }\end{array}$ \\
\hline
\end{tabular}

Fuente: http://www.iune.es/ 
Tabla II. Descripción de los indicadores de la actividad investigadora. Fuente Observatorio IUNE

\begin{tabular}{|c|c|}
\hline Etiquetas & Descripción \\
\hline P_PROF_PROM & Número de profesores por universidad (promedio) \\
\hline R_SEX_100PROF & Número de sexenios obtenidos por universidad por cada 100 profesores (promedio) \\
\hline AC_PUBL_PROME & Publicaciones por universidad (promedio) \\
\hline AC_PUBXPROF_PROMD & Publicaciones por profesor y universidad (promedio) \\
\hline AC_COAUTO_PROM & Autores por documento de la producción científica de cada universidad cada año (promedio) \\
\hline AC_COLABINTER_PROM & $\begin{array}{l}\text { Porcentaje de documentos firmados por cada universidad con, al menos, una institución } \\
\text { extranjera, respecto al total de documentos firmados por cada universidad cada año (promedio) }\end{array}$ \\
\hline AC_CITAS_TOT & $\begin{array}{l}\text { Mide el número de citas recibidas por la producción científica anual de cada universidad } \\
\text { (promedio) }\end{array}$ \\
\hline AC_CITASPROF_PROM & Ídem por profesores \\
\hline AC_DOCNC_PROM & $\begin{array}{l}\text { Porcentaje de documentos por universidad y año, que desde su publicación hasta el momento de } \\
\text { actualización de los datos, no han recibido ninguna cita (promedio) }\end{array}$ \\
\hline AC_1C_S_TOTAL & Porcentajes de publicaciones en revistas del 1er cuartil (total) \\
\hline AC_1C_PROM & Porcentajes de publicaciones en revistas del 1er cuartil (promedio) \\
\hline AC_TOP3_TOT & Publicaciones en las 3 primeras revistas de cada una de las áreas científicas (total) \\
\hline AC_TOP3_\%TOT & $\begin{array}{l}\text { Porcentaje de publicaciones en las } 3 \text { primeras revistas de cada una de las áreas científicas } \\
\text { (promedio) }\end{array}$ \\
\hline IN_PATnac_TOT & Número de patentes nacionales (total) \\
\hline IN_PATnacX100P_TOT & Número de patentes nacionales por cada 100 profesores (promedio) \\
\hline IN_ConI_DCONS_TOT & Importe de los contratos de I+D y consultorías (total miles euros) \\
\hline $\begin{array}{l}\text { IN_ConIDCONSX100MILES_EUROS_ } \\
\text { PROM }\end{array}$ & Ídem por cada 100 profesores (promedio \\
\hline IN_PSERVIC_MILES_EUROS & Importe facturado por prestación de servicios (total miles de euros) \\
\hline IN_PSERVICX100PMILES_EUROS_PROM & Ídem por cada 100 profesores (promedio \\
\hline IN_INGLICMILES_EUROS_PROM & $\begin{array}{l}\text { Importe generado por las actividades de uso, explotación, modificación, etc, (bajo unas } \\
\text { determinadas condiciones, según se acuerde en el contrato de licencia), de una determinada } \\
\text { tecnología o conocimiento de la universidad (total miles euros) }\end{array}$ \\
\hline IN_INGLICXPMILES_EUROS_PROM & Ídem por cada 100 profesores (promedio \\
\hline IN_PCTMILES_EUROS_PROM & $\begin{array}{l}\text { Número de protecciones de invenciones de forma simultánea en distintos países, a través de la } \\
\text { presentación de una solicitud internacional de patentes }\end{array}$ \\
\hline IN_PCTX100PMILES_EUROS_PROM & Ídem por cada 100 profesores (promedio) \\
\hline IN_SPINOFF_TOT & Número de spin-off creadas (total) \\
\hline IN_SPINOFFx100P_PROM & Ídem por cada 100 profesores (promedio) \\
\hline COM_PLANN_TOT & Número de proyectos obtenidos en convocatorias del Plan Nacional(total) \\
\hline COM_PLANN_PROM & Número de proyectos obtenidos en convocatorias del Plan Nacional(promedio) \\
\hline COM_PLANNX100P_TOT & $\begin{array}{l}\text { Número de proyectos obtenidos en convocatorias del Plan Nacional por cada } 100 \\
\text { profesores(promedio) }\end{array}$ \\
\hline COM_PMARCO_TOT & Número de proyectos obtenidos en convocatorias del Programas Marco (total) \\
\hline COM_PMARCO_PROM & Número de proyectos obtenidos en convocatorias del Programas Marco (promedio) \\
\hline COM_PMARCOX100P_PROM & $\begin{array}{l}\text { Número de proyectos obtenidos en convocatorias del Programas Marco por cada } 100 \text { profesores } \\
\text { (promedio) }\end{array}$ \\
\hline CF_FPI_PROM & Número de becas FPI (promedio) \\
\hline CF_FPIX100P_PROM & Número de becas FPI por cada 100 profesores (promedio) \\
\hline CF_FPU_PROM & Número de becas FPU (promedio) \\
\hline CF_FPUx100P_PROM & Número de becas FPU por cada 100 profesores (promedio) \\
\hline CF_JCIERVA_PROM & $\begin{array}{l}\text { Número de contratos del Programa Juan de la Cierva (contratos de doctores -3 años- para } \\
\text { incorporación a equipos de investigación) por universidad (promedio) }\end{array}$ \\
\hline CF_JCIERVAX100P_PROM & Ídem por cada 100 profesores y universidad (promedio) \\
\hline CF_RyCAJAL_PROM & $\begin{array}{l}\text { Número de contratos del Programa Ramón y Cajal (contrato laboral doctores -5 años- para } \\
\text { incorporación a equipos de investigación) por universidad (promedio) }\end{array}$ \\
\hline CF_RYCAJALX100P_PROM & Ídem por cada 100 profesores y universidad (promedio) \\
\hline CF_TESIS_PROM & Tesis doctorales defendidas por universidad (promedio) \\
\hline CF_TESIS $\times 100 P \_P R O M$ & Tesis doctorales defendidas por cada 100 profesores y universidad (promedio) \\
\hline
\end{tabular}




\section{IMPORTANCIA DE LA ANTIGÜEDAD Y DEL TAMAÑO DE LA UNIVERSIDAD EN SU ACTIVIDAD INVESTIGADORA}

La antigüedad y el tamaño de la universidad son factores que pueden incidir en la actividad investigadora. Universidades con más historia pueden contar con más infraestructuras y equipamiento, con más tradición y experiencia y con equipos más consolidados a lo largo del tiempo de funcionamiento. Obviamente, las universidades de mayor tamaño tienen más recursos materiales y humanos y cabe esperar que sea mayor también la actividad investigadora desarrollada en valor absoluto e incluso un efecto de economía de escala, pero no es tan evidente que esta situación se mantenga cuando la actividad investigadora se relativiza por algún indicador de tamaño como el número de profesores.

Entre las universidades españolas hay perfiles de actividad investigadora diversos por áreas temáticas (Bordons y otros, 2010), hay diversidad en la producción científica (Urdín y Morillo 2000) y en relaciones de colaboración científica (García y otros, 2012). Y, por supuesto, hay diversidad en la antigüedad de la universidad (desde casi ocho siglos a algo más de una década) y en el tamaño.

Se propone someter a contrastación, tomando como período de análisis la primera década del siglo XXI, la hipótesis de que las universidades con más antigüedad presentan mejores indicadores de actividad investigadora que las recientes. Esperando que las universidades más antiguas respecto a las más recientes tengan:

- H1a: Mayor actividad científica (producción, colaboración, impacto y visibilidad) tanto en valores absolutos como relativos por el número de profesores.

- H1b: Mejores indicadores de innovación tanto en valores absolutos como relativos por el número de profesores.

- H1c: Mayor competitividad tanto en valores absolutos como relativos por el número de profesores.

- H1d: Mayor capacidad formativa tanto en valores absolutos como relativos por el número de profesores.

Es una conclusión bastante evidente que el tamaño de la universidad resulte significativo para las diferencias en los indicadores absolutos de la actividad investigadora. No obstante, su consideración ahora es para ratificar esa influencia que podría ser anulada por otros factores. Es decir, una gran diferencia en recursos económicos entre universidades o en el ratio estudiantes/profesor podrían reducir o anular las diferencias en los indicadores de actividad científica imputables al tamaño (número de profesores). En suma, por un lado, se pretende comprobar si hay diferencia entre las formas de considerar el tamaño, en nuestro caso, según número de profesores y según número de estudiantes $y$, por otro lado, se persigue comprobar en qué indicadores de la actividad investigadora la influencia del tamaño de la universidad es mayor, es decir, qué indicadores son más sensibles a la influencia del tamaño.

Así, según las hipótesis relativas al tamaño $(\mathrm{H} 2)$, medido por número de profesores y por número de estudiantes, cabe esperar que las universidades más grandes, tanto por profesores como por estudiantes, tengan mayores indicadores que las menos grandes en:

- H2a: Mayor actividad científica (producción, colaboración, impacto y visibilidad) tanto en valores absolutos como relativos por el número de profesores.

- H2b: Mejores indicadores de innovación tanto en valores absolutos como relativos por el número de profesores.

- H2c: Mayor competitividad tanto en valores absolutos como relativos por el número de profesores.

- H2d: Mayor capacidad formativa tanto en valores absolutos como relativos por el número de profesores.

Respecto al ratio estudiantes por profesor $(\mathrm{H} 3)$, cabe esperar que un menor ratio suponga una mayor posibilidad de dedicar esfuerzo a la actividad investigadora $y$, por tanto, las universidades con un menor ratio se espera que presenten:

- H3a: Mayor actividad científica (producción, colaboración, impacto y visibilidad) tanto en valores absolutos como relativos por el número de profesores.

- H3b: Mejores indicadores de innovación tanto en valores absolutos como relativos por el número de profesores.

- H3c: Mayor competitividad tanto en valores absolutos como relativos por el número de profesores.

- H3d: Mayor capacidad formativa tanto en valores absolutos como relativos por el número de profesores.

\section{METODOLOGÍA}

Los datos se obtienen del Observatorio IUNE que; como indica en su web (http://www.iune.es/), y ya se ha comentado, es una herramienta que permite conocer los resultados más significativos de la actividad investigadora de las universidades españolas públicas y privadas. Comprende un conjunto de indicadores agrupados por diferentes aspectos de la actividad universitaria: profesorado, reconocimiento, actividad científica, innovación, competitividad 
y capacidad formativa. Actualmente se presenta la información relativa al período 2002-2010, que es el período que se analiza ahora. Este observatorio se alimenta de datos de fuentes oficiales, contrastadas y fiables.

Además se utilizan las variables: "Número de estudiantes por profesor", "Número de estudiantes matriculados" y "Número de estudiantes equivalente a tiempo completo". Estas variables se han tomado de los datos de la universidad en cifras (Hernández Armenteros y otros, 2010; Hernández Armenteros, 2008; Hernández Armenteros, 2006; Hernández Armenteros, 2004; Hernández Armenteros 2002) y la media para la década se ha calculado haciendo la media de esos cinco informes lo que, se asume, constituye una buena estimación de la media de la primera década del siglo XXI.

El conjunto objeto de estudio está formado por las universidades públicas presenciales españolas que son 47. Para cada variable independiente se ha calculado la mediana para dividir en dos la población, para después aplicar el análisis de la varianza y el test de Mann-Whitney para contrastar la existencia de diferencias significativas por la antigüedad y tamaño. Posteriormente se ha efectuado análisis de regresión lineal tomando las variables de la actividad investigadora como dependientes y el tamaño, antigüedad y ratio estudiantes-profesor como independientes para ver su influencia.

\section{ANÁLISIS DE DATOS}

\section{La antigüedad de las universidades y la actividad investigadora}

La variable antigüedad recoge el número de años desde la fundación de la universidad. Utilizando la mediana, el conjunto de universidades se ha dividido en dos: universidades fundadas hasta 1978 (23 universidades) y universidades fundadas en ese año y con posterioridad (24 universidades), cuyo detalle se puede ver en la tabla III. Se trata de un año de la transición política española, con un nuevo período inaugurado con la Constitución de 1978 y justo antes del desarrollo del estado de las autonomías. En definitiva, el valor de la mediana coincide con un año especial y pertinente por razones del contexto social y político.

Para comprobar la existencia de diferencias significativas entre universidades antiguas y recientes, se aplica análisis de la varianza y el test de MannWhitney, que resultan en gran medida coincidentes, a los valores promedio o totales de indicadores de la actividad investigadora. Efectivamente, se constatan numerosas e importantes diferencias significativas a favor de las universidades con más años que triplican, y a veces sobradamente, a los valores de las universidades más recientes (Tabla IV).

Para empezar, la antigüedad de las universidades públicas españolas está correlacionada de forma importante con el tamaño $(0,671)$, esto es, a más antigüedad también más tamaño. Las universidades más antiguas son más grandes, tienen más del doble de estudiantes en total o estudiantes a tiempo completo (en concreto 2,46 veces más) pero sobre todo tienen un mayor número de profesores, el triple. Sin embargo, no hay diferencias en el número de sexenios por cada 100 profesores.

La actividad científica de las universidades más antiguas se caracteriza respecto a las universidades más recientes por tener más producción (el triple de artículos durante el período considerado) y mayor colaboración con otras por el número de coautorías (aunque en menor medida, solamente 1,61 veces). El impacto de la investigación también es mayor por el número de citas totales recibidas $(3,31$ veces $)$ y en visibilidad tanto por contar con un porcentaje de artículos sobre el total mayor, como por el número de artículos de las top 3. En ambos casos las universidades antiguas superan en algo más de 3 veces a las recientes, igual que en los casos que siguen según se muestra en la tabla IV. Sin embargo, en otros indicadores en valores absolutos no se producen diferencias significativas, como es el caso del número de colaboraciones internacionales o el porcentaje de trabajos en publicaciones top.

En cuanto a los indicadores de innovación, también las universidades antiguas presentan magnitudes que triplican sobradamente el número total de patentes, el importe obtenido por contratos de I $+D$ y consultorías, los ingresos por actividades de uso de explotación, modificación, etc. de una determinada tecnología o conocimiento, o ya sea por el número medio de spin-off creadas que triplica al de las universidades más nuevas.

En los valores de competitividad, las universidades más antiguas consiguen un número de proyectos de investigación en el Plan Nacional y en los Programas Marco mayor que el promedio de las universidades más recientes, en aproximadamente el triple.

Respecto a la última dimensión, la capacidad formativa, de nuevo existen diferencias importantes a favor de las antiguas en el número de becas FPI, FPU (la mayor diferencia con 5,85 veces más); número de contratos tanto en la modalidad Juan de la Cierva como Ramón y Cajal. El número medio de tesis leídas en las universidades antiguas cuadruplica el promedio de las universidades nuevas.

Por el contrario, cuando las magnitudes se relativizan por el número de profesores (por cada 100 profesores) las diferencias destacables se presentan en menos indicadores y tienen un carácter más bien excepcional. Así, no hay diferencias significativas en el reconocimiento de sexenios por profesor entre las universidades, ni en las de producción, innovación o competitividad. Solamente es en la capacidad formativa donde se producen diferencias significativas entre antiguas y nuevas al relativizar 
por el número de profesores. En concreto, para el número de becas FPU puesto que hay más becas de este tipo por cada 100 profesores en las universidades antiguas (1,69 veces) y, especialmente, en el número de tesis defendidas con 1,29 veces más que en las universidades más recientes.

En suma, se confirman las distintas variantes de la hipótesis $\mathrm{H} 1$ para los valores absolutos de los indicadores de la actividad investigadora, pero no se confirman para los valores relativos salvo las dos excepciones referidas.

\section{El tamaño de las universidades, medido por número de profesores, y la actividad investi- gadora}

Para analizar el tamaño se tuvo en cuenta, en primer lugar, el número medio de profesores durante el período analizado. Se utilizó la mediana para obtener dos grupos de universidades, las que tienen mayor y menor número de profesores ( 24 y 23 universidades, respectivamente), según se detalla en la tabla III, porque están por encima o debajo de la mediana, procediendo lo mismo que en el caso anterior.

Tabla III. Relación de universidades que componen los grupos separados por la mediana para las variables antigüedad, tamaño (por número de profesores) y ratio estudiantes-profesor

\begin{tabular}{|c|c|c|c|c|c|c|c|c|c|c|c|}
\hline \multicolumn{4}{|c|}{$\begin{array}{l}\text { Antigüedad } \\
\text { Me:34 }\end{array}$} & \multicolumn{4}{|c|}{$\begin{array}{c}\text { Número medio de profesores } \\
\text { Me:938 }\end{array}$} & \multicolumn{4}{|c|}{$\begin{array}{c}\text { Ratio estudiantes/profesor } \\
\text { Me:15,91 }\end{array}$} \\
\hline \multicolumn{2}{|l|}{ G1 } & \multicolumn{2}{|c|}{ G2 } & \multicolumn{2}{|l|}{ G1 } & \multicolumn{2}{|c|}{ G2 } & \multicolumn{2}{|l|}{ G1 } & \multicolumn{2}{|c|}{ G2 } \\
\hline Univer. & Años & Univer. & Años & Univer. & No & Univer. & No & Univer. & No & Univer. & No \\
\hline UIB & 34 & USAL & 794 & UCA & 884 & UCM & 3816 & UCO & 15,8 & UPO & 37,3 \\
\hline UA & 33 & UCM & 719 & UDC & 839 & UB & 2687 & UCA & 15,8 & URJC & 28,9 \\
\hline UCA & 33 & UVA & 717 & UAH & 830 & UPM & 2481 & UAM & 15,5 & UPF & 25,2 \\
\hline UNILEON & 33 & UB & 582 & UCO & 803 & EHU & 2474 & UDG & 15,4 & UC3M & 24,6 \\
\hline UCLM & 30 & UNIZAR & 538 & UNICAN & 639 & US & 2398 & USAL & 15,2 & UNEX & 21,3 \\
\hline UNAVARRA & 25 & USC & 517 & UNILEON & 622 & UV & 2248 & UAH & 15,2 & UJI & 21,2 \\
\hline ULPGC & 23 & UV & 512 & URJC & 622 & UGR & 2246 & UNAVARRA & 15,1 & UCLM & 20,9 \\
\hline UC3M & 23 & US & 507 & UJAEN & 575 & UPV & 1750 & EHU & 14,6 & UA & 20,9 \\
\hline UDC & 23 & UGR & 481 & UIB & 524 & UNIZAR & 1725 & UNIRIOJA & 14,5 & UDC & 20,6 \\
\hline UVIGO & 23 & UNIOVI & 404 & UAL & 513 & USC & 1545 & UPV & 14,5 & US & 19,4 \\
\hline UPF & 22 & ULL & 220 & URV & 509 & UNIOVI & 1491 & USC & 14,5 & UHU & 18,7 \\
\hline UDG & 21 & UM & 97 & UC3M & 473 & UAB & 1446 & UVA & 14,2 & UJAEN & 18,4 \\
\hline UDL & 21 & EHU & 44 & UJI & 456 & UAM & 1444 & UIB & 14,1 & UGR & 18,3 \\
\hline UJI & 21 & UAB & 44 & UDG & 438 & UVA & 1443 & UPCT & 13,9 & UVIGO & 17,9 \\
\hline URV & 21 & UAM & 44 & UDL & 424 & UPC & 1388 & UNICAN & 13,2 & UM & 17,7 \\
\hline UNIRIOJA & 20 & UPC & 41 & UHU & 419 & USAL & 1366 & UPC & 13,2 & UNILEON & 17,1 \\
\hline UAL & 19 & UPM & 41 & UNAVARRA & 384 & UMA & 1352 & UB & 12,9 & ULPGC & 16,9 \\
\hline UHU & 19 & UPV & 41 & UBU & 376 & ULL & 1311 & UNIOVI & 12,9 & URV & 16,9 \\
\hline UJAEN & 19 & UCO & 40 & UMH & 349 & UM & 1235 & UNIZAR & 12,2 & UCM & 16,7 \\
\hline UBU & 18 & UMA & 40 & UPCT & 293 & UNEX & 1013 & UPM & 11,7 & UAB & 16,6 \\
\hline UMH & 16 & UNICAN & 40 & UPF & 285 & UA & 990 & UDL & 11,3 & UAL & 16,5 \\
\hline URJC & 16 & UNEX & 39 & UNIRIOJA & 263 & UCLM & 980 & ULL & 11,2 & UBU & 16,2 \\
\hline UPO & 14 & UAH & 35 & UPO & 184 & UVIGO & 952 & UMA* & & UV & 15,9 \\
\hline UPCT & 14 & & & & & ULPGC & 938 & UMH * & & & \\
\hline
\end{tabular}

$\left(^{*}\right)$ Casos para los que no se dispone de datos 
En términos generales, lo ya comentado se mantiene. Las diferencias significativas en la actividad investigadora entre universidades con más y con menos años ahora se presentan al comparar las que tienen más con las que tienen menos profesores (Tabla IV). Se detectan además algunas peculiaridades:

- Hay nuevas diferencias entre grupos como la cifra de ingresos por prestación de servicios es mayor en las universidades mayores $(6,52$ veces) con diferencias significativas en valores absolutos y cuasi significativas en el caso de relativizar por cada 100 profesores.

- Diferencias que se suavizan, son algo menos significativas: la colaboración por coautorías pasa de ser significativa o una diferencia cuasi significativa $(0,08)$, siendo mayores en las universidades con más profesores. Ahora la diferencia entre universidades en cuanto al número de becas FPU por cada 100 profesores pasa a ser cuasi significativa $(0,07)$.

- Hay diferencias que desaparecen. No hay diferencias significativas en el número de tesis defendidas por cada 100 profesores.

En definitiva, para los valores absolutos de los indicadores de la actividad investigadora se cumplen todas las variantes de la hipótesis H2. Para los valores relativos, se rechazan todas las variantes de $\mathrm{H} 2$, con la excepción de las becas FPU por cada 100 profesores que resulta cuasi-significativa.

La incidencia del tamaño se da, de manera especial a favor de las más grandes, en los indicadores absolutos de ingresos por contratos de $I+D$, ingresos por licencias, ingresos por prestación de servicios, número de becas FPU o número de tesis defendidas. En estos casos el promedio del grupo de las grandes cuadruplica al promedio del grupo de las universidades menores. Tampoco ahora se producen diferencias significativas en el número de colaboraciones internacionales o el porcentaje de trabajos en publicaciones top.

\section{El tamaño de las universidades, medido por el número de estudiantes matriculados y núme- ro de estudiantes equivalentes a tiempo com- pleto, y la actividad investigadora}

La correlación del número de estudiantes con el número de estudiantes a tiempo completo es casi total y ambas variables están muy correlacionadas con el número de profesores $(0,965$ y 0.954$)$ y en menor medida, aunque sigue siendo eleva$\mathrm{da}$, con la antigüedad (0,64 aproximadamente, en ambos casos). Por tanto, operando con la mediana tanto para el número de estudiantes matriculados (mediana igual a 20.715) como para el número de estudiantes equivalentes a tiempo completo (con una mediana de 17.025), la interpretación coincide con lo ya comentado para el número de profesores. Hay que advertir algún cambio respecto al núme- ro de profesores. No hay diferencias, ni si quiera cuasi significativas, en los ingresos por prestación de servicios por cada 100 profesores mientras que aparecen diferencias más fuertes en el número de becas FPU por cada 100 profesores, siendo claramente mayor el número de estas becas en el grupo de universidades con un mayor promedio de estudiantes matriculados.

\section{La ratio número de estudiantes/profesor y la actividad investigadora}

Esta ratio se refiere al promedio del número de estudiantes matriculados por profesor en el periodo 2001-2010 obtenidos de las diferentes publicaciones de "La universidad en cifras" dirigida por el profesor Hernández Armenteros. Tiene una correlación negativa $(-0,317)$ y estadísticamente significativa con el tamaño, es decir, a mayor número medio de profesores menor es el promedio de la ratio estudiantes-profesor. Recurriendo a la mediana se discriminó entre dos grupos de universidades, las que tienen una ratio media menor a 15,9 frente a las que tienen una ratio estudiantes por profesor mayor, según se detalla en la tabla III.

En este caso la situación es muy diferente porque son escasos los indicadores que presentan diferencias importantes, ni en los valores absolutos ni en los valores relativos por profesores.

Con frecuencia, el grupo de universidades con mayor ratio estudiantes-profesor presenta mejores indicadores relativizados por el número de profesores y lo contrario sucede con los indicadores en valores absolutos, pero son excepciones los casos en los que tales diferencias son estadísticamente significativas.

El caso más claro de diferencia es el de los ingresos por explotación, modificación, etc. (bajo unas determinadas condiciones, según se acuerde en el contrato de licencia), de una determinada tecnología o conocimiento de la universidad en valor absoluto, mucho mayor en el grupo de universidades con menor ratio de estudiantes/profesor. Para el resto de excepciones las diferencias son cuasi significativas (niveles entre el $5 \%$ y el $10 \%$ ) y las hay para el número de patentes nacionales, número de becas FPI y número de becas FPU, en estos casos las universidades con menor ratio estudiantesprofesor superan claramente a las de mayor ratio. Cuando se relativiza por cada 100 profesores, hay diferencia para el número de proyectos de Programas Marco (es mayor en el grupo de universidades con mayor ratio de estudiantes-profesor) y para los ingresos por explotación, modificación, etc. (bajo unas determinadas condiciones, según se acuerde en el contrato de licencia), de una determinada tecnología o conocimiento.

Es de destacar que las diferencias entre universidades por la ratio de estudiantes-profesor son muchos menores que las diferencias existentes por número de profesores, antigüedad o número de estudiantes. 
Tabla IV. Diferencias en los indicadores de la actividad investigadora según antigüedad y tamaño (por número de profesores y número de estudiantes) y ratio estudiantes-profesor (las líneas sombreadas corresponden a indicadores referidos a cada 100 profesores)

\begin{tabular}{|c|c|c|c|c|c|c|c|c|}
\hline \multirow{2}{*}{ INDICADORES } & \multicolumn{2}{|c|}{ PROFESORES } & \multicolumn{2}{|c|}{ ANTIGÜEDAD } & \multicolumn{2}{|c|}{$\begin{array}{l}\text { ESTUDIANTES } \\
\text { MATRICULADOS }\end{array}$} & \multicolumn{2}{|c|}{$\begin{array}{c}\text { ESTUDIANTES/ } \\
\text { PROFESOR } \\
\end{array}$} \\
\hline & Sig.* & $\begin{array}{c}\text { Ratio** } \\
\text { G2/G1 }\end{array}$ & Sig.* & $\begin{array}{c}\text { Ratio** } \\
\text { G2/G1 }\end{array}$ & Sig.* & $\begin{array}{l}\text { Ratio** } \\
\text { G2/G1 }\end{array}$ & Sig.* & $\begin{array}{c}\text { Ratio** } \\
\text { G2/G1 }\end{array}$ \\
\hline R_SEX_100PROF & 0,34 & 0,90 & 0,57 & 0,93 & 0,34 & 0,91 & 0,26 & 1,11 \\
\hline AC_PUBL_PROME & 0,00 & 3,20 & 0,00 & 3,07 & 0,00 & 3,09 & 0,27 & 0,83 \\
\hline AC_PUBXPROF_PROMD & 0,56 & 0,91 & 0,91 & 0,95 & 0,40 & 0,90 & 0,96 & 1,10 \\
\hline AC_COAUTO_PROM & 0,08 & 1,50 & 0,00 & 1,61 & 0,07 & 1,53 & 0,17 & 0,76 \\
\hline AC_COLABINTER_PROM & 0,33 & 1,05 & 0,16 & 1,06 & 0,23 & 1,06 & 0,29 & 0,96 \\
\hline AC_CITAS_TOT & 0,00 & 3,48 & 0,00 & 3,31 & 0,00 & 3,34 & 0,29 & 0,78 \\
\hline AC_CITASPROF_PROM & 0,79 & 0,95 & 0,66 & 0,98 & 0,98 & 0,94 & 0,98 & 1,09 \\
\hline AC_DOCNC_PROM & 0,93 & 1,01 & 0,72 & 1,01 & 0,92 & 1,02 & 0,11 & 1,05 \\
\hline AC_1C_S_TOTAL & 0,00 & 3,28 & 0,00 & 3,24 & 0,00 & 3,17 & 0,15 & 0,79 \\
\hline AC_1C_PROM & 0,83 & 1,01 & 0,39 & 1,03 & 0,70 & 1,01 & 0,61 & 0,98 \\
\hline AC_TOP3_TOT & 0,00 & 3,05 & 0,00 & 3,13 & 0,00 & 2,99 & 0,31 & 0,81 \\
\hline AC_TOP3_\%TOT & 0,66 & 0,97 & 0,60 & 1,02 & 0,84 & 0,97 & 0,32 & 1,06 \\
\hline IN_PATnac_TOT & 0,00 & 3,13 & 0,00 & 3,32 & 0,00 & 2,91 & 0,10 & 0,62 \\
\hline IN_PATnacX100P_TOT & 0,73 & 1,01 & 0,59 & 1,18 & 0,46 & 0,96 & 0,20 & 0,73 \\
\hline IN_ConI_DCONS_TOT & 0,00 & 4,02 & 0,00 & 4,18 & 0,00 & 3,86 & 0,45 & 0,54 \\
\hline IN_ConIDCONSx100MILES_EUROS_PROM & 0,87 & 1,02 & 0,68 & 1,17 & 0,98 & 1,02 & 0,79 & 0,94 \\
\hline IN_PSERVIC_MILES_EUROS & 0,00 & 6,52 & 0,02 & 5,71 & 0,00 & 6,20 & 0,96 & 0,64 \\
\hline IN_PSERVICx100PMILES_EUROS_PROM & 0,08 & 2,78 & 0,21 & 2,61 & 0,11 & 2,81 & 0,64 & 0,75 \\
\hline IN_INGLICMILES_EUROS_PROM & 0,01 & 4,61 & 0,01 & 3,58 & 0,00 & 5,07 & 0,01 & 0,34 \\
\hline IN_INGLICXPMILES_EUROS_PROM & 0,96 & 1,04 & 0,70 & 1,00 & 0,63 & 1,15 & 0,09 & 0,65 \\
\hline IN_PCTMILES_EUROS_PROM & 0,00 & 3,54 & 0,00 & 3,63 & 0,00 & 3,75 & 0,39 & 0,59 \\
\hline IN_PCTX100PMILES_EUROS_PROM & 0,21 & 1,22 & 0,14 & 1,33 & 0,13 & 1,29 & 0,63 & 0,88 \\
\hline IN_SPINOFF_TOT & 0,00 & 3,02 & 0,00 & 3,31 & 0,01 & 2,83 & 0,11 & 0,57 \\
\hline IN_SPINOFFx100P_PROM & 0,30 & 0,87 & 0,92 & 1,13 & 0,22 & 0,86 & 0,48 & 0,79 \\
\hline COM_PLANN_TOT & 0,00 & 2,96 & 0,00 & 3,00 & 0,00 & 2,94 & 0,15 & 0,77 \\
\hline COM_PLANN_PROM & 0,00 & 2,96 & 0,00 & 3,00 & 0,00 & 2,94 & 0,15 & 0,77 \\
\hline COM_PLANNx100P_TOT & 0,12 & 0,79 & 0,43 & 0,86 & 0,11 & 0,80 & 0,53 & 1,06 \\
\hline COM_PMARCO_TOT & 0,00 & 2,95 & 0,00 & 3,09 & 0,00 & 3,02 & 0,14 & 0,59 \\
\hline COM_PMARCO_PROM & 0,00 & 2,83 & 0,00 & 2,97 & 0,00 & 2,91 & 0,11 & 0,59 \\
\hline COM_PMARCOX100P_PROM & 0,68 & 0,75 & 0,91 & 0,84 & 0,70 & 0,78 & 0,09 & 1,10 \\
\hline CF_FPI_PROM & 0,00 & 3,36 & 0,00 & 3,70 & 0,00 & 3,56 & 0,08 & 0,69 \\
\hline CF_FPIx100P_PROM & 0,93 & 0,88 & 0,17 & 1,04 & 0,59 & 0,94 & 0,18 & 0,95 \\
\hline CF_FPU_PROM & 0,00 & 4,83 & 0,00 & 5,85 & 0,00 & 5,20 & 0,08 & 0,80 \\
\hline CF_FPUx100P_PROM & 0,07 & 1,34 & 0,00 & 1,69 & 0,03 & 1,44 & 0,36 & 0,92 \\
\hline CF_JCIERVA_PROM & 0,00 & 3,05 & 0,00 & 2,99 & 0,00 & 3,27 & 0,62 & 0,95 \\
\hline CF_JCIERVAx100P_PROM & 0,83 & 0,77 & 0,57 & 0,81 & 0,49 & 0,83 & 0,60 & 1,59 \\
\hline CF_RyCAJAL_PROM & 0,00 & 3,04 & 0,00 & 3,62 & 0,00 & 3,23 & 0,21 & 0,83 \\
\hline CF_RyCAJALx100P_PROM & 0,78 & 0,75 & 0,23 & 0,95 & 0,91 & 0,80 & 0,72 & 1,19 \\
\hline CF_TESIS_PROM & 0,00 & 3,99 & 0,00 & 4,25 & 0,00 & 4,01 & 0,25 & 0,83 \\
\hline CF_TESIS X100P_PROM & 0,21 & 1,14 & 0,01 & 1,29 & 0,15 & 1,16 & 0,98 & 1,01 \\
\hline
\end{tabular}

*Nivel de significación del test Mann-Whitney.

**Cociente entre el grupo de universidades mayor (por tamaño, antigüedad, número de estudiantes o ratio estudiantes-profesor) y el grupo menor. 
En términos generales, y con las salvedades mencionadas, se rechaza la $\mathrm{H} 3$ en sus distintas variantes, tanto para los valores absolutos como para los relativos por número de profesores.

\section{Consideración conjunta del tamaño, antigüe- dad y ratio estudiantes-profesor}

Interesa conocer la influencia de las tres variables (tamaño, antigüedad y ratio estudiantes-profesor) conjuntamente sobre los indicadores de la actividad investigadora, identificar en qué indicadores su influencia es significativa y cuantificar la importancia de la misma.

Para ello aplicamos un modelo de regresión lineal como la técnica apropiada para observar los efectos principales de estas variables en esta situación. En términos generales, los mayores ajustes (ver $\mathrm{R}^{2}$ ), es decir cuando las variables independientes explican mejor los indicadores de la actividad investigadora, se presentan para los indicadores expresados en valores absolutos (totales o promedio) Es el caso del número de proyectos conseguidos en planes nacionales, el número de tesis defendidas, el número de publicaciones promedio y el número de becas FPU con un $\mathrm{R}^{2}$ que supera el 0,7 . Están cerca de ese nivel el número promedio de becas $\mathrm{FPI}$, el número de total de artículos en el primer cuartil, número de artículos en el top 3 y el promedio de citas.

Mientras que para los indicadores relativizados por el número de profesores no hay ajustes aceptables con la salvedad del número de sexenios y los ingresos por prestación de servicios, con un $\mathrm{R}^{2}$ de 0,31 y 0,34 , respectivamente (Tabla V).

\section{- Tamaño (número de profesores)}

El número de profesores es la variable que contribuye a esos ajustes en más casos y con mayor fuerza en todos los indicadores en los que resulta significativo. Principalmente esto ocurre para el número de proyectos del Plan Nacional, número de proyectos del Programa Marco, el número de tesis defendidas, el número de publicaciones, el número de artículos en el primer cuartil, el número de becas FPI, importe de los contratos de I+D y consultorías, importe facturado por prestación de servicios, número de protecciones de invenciones de una solicitud internacional de patentes y número de contratos Juan de la Cierva. Para todos estos casos el coeficiente beta es igual o superior a 0,8. Así, un profesor más, como promedio en las universidades, ha supuesto 0,5 artículos más; 0,15 tesis defendidas de promedio más, 0,37 proyectos del Plan Nacional más o 65 mil euros más de ingresos en el total del período considerado.

También es destacable la incidencia del número de profesores (puesto que supera el 0,7 de coeficiente beta) en el número de citas totales, artículos en el top 3 y de contratos Ramón y Cajal y también aunque algo menos en el número de spin-off.

Cuando los indicadores están relativizados por el número de profesores, solamente hay un caso en el que el tamaño tiene un efecto significativo. Es en el importe facturado por prestación de servicios. De manera que contar con un profesor más de promedio durante el período supone un mayor importe por prestación de servicios de 0,126 mil euros por cada 100 profesores.

\section{- Antigüedad}

Respecto a la antigüedad, su importancia se manifiesta en un tercio de los casos y siempre es menor que la del tamaño, de hecho cuando se considera el tamaño como covariable en un análisis de la covarianza (Luque-Martínez, 2012), en general el efecto de la antigüedad desaparece. Es de destacar su relación positiva con el número de becas FPU y, sobre todo, la relación negativa respecto al número de proyectos del Programa Marco y la mayor parte de los indicadores de innovación como los ingresos por contratos de I+D y consultorías (tanto en valor absoluto como relativo), ingresos por prestación de servicios (en valor absoluto y relativo), ingresos por patentes y el número de spin-off. De manera que a más antigüedad, mayor número de becas FPU pero menor número de proyectos del Programa Marco, menor número de spin-off y menores ingresos por I+D y consultorías, prestación de servicios o de patentes.

Para el caso de indicadores por cada 100 profesores, se repite la situación respecto a algunos indicadores de innovación. Así, hay una relación negativa entre antigüedad y los ingresos por I+D y consultorías y prestación de servicios por profesor.

En todas estas situaciones en las que resulta significativo el número de años de la universidad, también lo es el tamaño (número de profesores) y con más fuerza. Por otro lado, las universidades más recientes están más orientadas a la innovación a la vista de esta relación negativa.

\section{- Ratio estudiantes profesor}

La ratio estudiantes por profesor contribuye a explicar, de manera exclusiva, y sin que resulte de importancia ni el tamaño ni la antigüedad, tres indicadores. Uno es de tipo absoluto, el número de documentos no citados, y dos son de tipo relativo: el número de sexenios por profesor y el número de proyectos de investigación conseguidos en el Plan Nacional.

En los tres casos mediante una relación positiva, esto es, a mayor ratio más proyectos y más sexenios. Llama la atención y es chocante que a mayor ratio de estudiantes-profesor, más proyectos del Plan Nacional y más sexenios. Esto se debe a que hay una serie de universidades muy eficaces para o con una orientación a la consecución de sexenios y de proyectos. Así, de las 15 universidades que superan el promedio de sexenios, 13 son de mayor ratio medio de estudiantes-profesor y de las seis primeras, cinco pertenecen a este grupo y son: UPO, UC3M, UPF, UJI y UAB. Respecto al promedio de proyectos del Plan Nacional de las siete primeras, cinco pertenecen al grupo de mayor ratio y las tres primeras superan el doble de la media total y son las mismas universidades, ahora por el siguiente orden: UPF, UPO, UC3M, UAB y UJI. 
Tabla V. Coeficientes B, Beta y $\mathrm{R}^{2}$ para las regresiones lineales utilizando como variables independientes el número de profesores, al número de años de antigüedad de la universidad y la ratio estudiantes-profesor (las líneas sombreadas corresponden a indicadores referidos a cada 100 profesores)

\begin{tabular}{|c|c|c|c|c|c|c|c|}
\hline \multirow[b]{2}{*}{ INDICADORES } & \multicolumn{2}{|c|}{ No profesores } & \multicolumn{2}{|c|}{ Antigüedad } & \multicolumn{2}{|c|}{ Estudiantes-profesor } & \multirow{2}{*}{$\mathbf{R}^{2}$} \\
\hline & B & Beta & B & Beta & B & Beta & \\
\hline R_SEX_100PROF & 0,000 & $-0,159$ & 0,002 & 0,169 & $0,243^{*}$ & $0,529 *$ & 0,31 \\
\hline AC_PUBL_PROME & $0,515^{*}$ & $0,857 *$ & 0,054 & 0,026 & 3,163 & 0,032 & 0,75 \\
\hline AC_PUBXPROF_PROMD & $-6,297 \mathrm{E}-6$ & $-0,023$ & $-1.566 \mathrm{E}-6$ & $-0,017$ & 0,009 & 0,202 & 0,05 \\
\hline AC_COAUTO_PROM & 0,001 & 0,187 & 0,000 & 0,005 & $-0,162$ & $-0,152$ & 0,08 \\
\hline AC_COLABINTER_PROM & 0,001 & 0,109 & 0,003 & 0,119 & $-0,011$ & $-0,010$ & 0,05 \\
\hline AC_CITAS_TOT & $42,643 *$ & $0,743 *$ & 10,246 & 0,053 & 209,785 & 0,022 & 0,60 \\
\hline AC_CITASPROF_PROM & 0,000 & 0,033 & $-2,067 \mathrm{E}-6$ & 0,000 & 0,082 & 0,136 & 0,02 \\
\hline AC_DOCNC_PROM & 0,00 & 0,097 & 0,001 & 0,046 & $0,230 * *$ & $0,392 * *$ & 0,14 \\
\hline AC_1C_S_TOTAL & $2,204 *$ & $0,806 *$ & 0,161 & 0,017 & 6,567 & 0,014 & 0,66 \\
\hline AC_1C_PROM & 0,001 & 0,092 & 0,000 & 0,012 & $-0,02$ & $-0,018$ & 0,01 \\
\hline AC_TOP3_TOT & $0,452 *$ & $0,791 *$ & $-0,009$ & $-0,005$ & 2,840 & 0,030 & 0,61 \\
\hline AC_TOP3_\%TOT & 0,000 & 0,145 & $-0,001$ & $-0,145$ & 0,084 & 0,206 & 0,05 \\
\hline IN_PATnac_TOT & $0.047 *$ & $0,761^{*}$ & $-0,061$ & $-0,291$ & $-1,395$ & $-0,135$ & 0,43 \\
\hline IN_PATnacX100P_TOT & $4,606 \mathrm{E}-5$ & 0,112 & 0,000 & $-0,310$ & $-0,018$ & $-0,266$ & 0,11 \\
\hline IN_COnI_DCONS_TOT & $65,720 *$ & $0,845^{*}$ & $-134,701^{*}$ & $-0,518^{*}$ & $-1363,5$ & $-0,108$ & 0,44 \\
\hline IN_ConIDCONSX100MILES_EUROS_PROM & 0,284 & 0,379 & $-1,258 * *$ & $-0,508 * *$ & 6,368 & 0,052 & 0,15 \\
\hline IN_PSERVIC_MILES_EUROS & $8,728^{*}$ & $0,841^{*}$ & $-14,931 *$ & $-0,431 *$ & $-230,523$ & $-0,132$ & 0,47 \\
\hline IN_PSERVICX100PMILES_EUROS_PROM & $0,127 *$ & $0,728 *$ & $-0,297 * *$ & $-0,51 * *$ & $-4,512$ & $-0,150$ & 0,34 \\
\hline IN_INGLICMILES_EUROS_PROM & $0,254 *$ & $0,468 *$ & $-0,716$ & $-0,401$ & $-15,658$ & $-0,179$ & 0,19 \\
\hline IN_INGLICXPMILES_EUROS_PROM & $-0,001$ & $-0,095$ & $-0,002$ & $-0,084$ & $-0,147$ & $-0,110$ & 0,03 \\
\hline IN_PCTMILES_EUROS_PROM & $0,027 *$ & $0,821 *$ & $-0,037 * *$ & $-0,34 * *$ & $-0,576$ & $-0,104$ & 0,47 \\
\hline IN_PCTX100PMILES_EUROS_PROM & 0,000 & 0,323 & 0,000 & $-0,215$ & 0,013 & 0,210 & 0,08 \\
\hline IN_SPINOFF_TOT & $0,013^{*}$ & $0,682 *$ & $-0,030 * *$ & $-0,48 * *$ & $-0,486$ & $-0,156$ & 0,32 \\
\hline IN_SPINOFFX100P_PROM & $-5,438 \mathrm{E}-6$ & $-0,019$ & 0,000 & $-0,305$ & $-0,010$ & $-0,222$ & 0,11 \\
\hline COM_PLANN_TOT & $0,372 *$ & $0,870 *$ & 0,066 & 0,045 & 1,157 & 0,016 & 0,80 \\
\hline COM_PLANN_PROM & $0,041^{*}$ & $0,870 *$ & 0,007 & 0,045 & 0,129 & 0,016 & 0,80 \\
\hline COM_PLANNX100P_TOT & 0,000 & $-0,176$ & 0,001 & 0,064 & $0,166 * *$ & $0,373 * *$ & 0,19 \\
\hline COM_PMARCO_TOT & $0,062 *$ & $0,819 *$ & $-0,111^{*}$ & $-0,438^{*}$ & $-1,425$ & $-0,114$ & 0,43 \\
\hline COM_PMARCO_PROM & $0,007 *$ & $0,818 *$ & $-0,012 *$ & $-0,443 *$ & $-0,161$ & $-0,117$ & 0,43 \\
\hline COM_PMARCOX100P_PROM & $5,435 \mathrm{E}-5$ & 0,065 & $-0,001$ & $-0,202$ & 0,017 & 0,123 & 0,05 \\
\hline CF_FPI_PROM & $0,012^{*}$ & $0,824 *$ & $-0,001$ & 0,017 & $-0,013$ & $-0,005$ & 0,67 \\
\hline CF_FPIX100P_PROM & $1,236 \mathrm{E}-5$ & 0,013 & $-1,412 \mathrm{E}-5$ & $-0,004$ & 0,025 & 0,153 & 0,02 \\
\hline CF_FPU_PROM & $0,05^{*}$ & $0,675 *$ & $0,066 * *$ & $0,265^{* *}$ & 0,712 & 0,058 & 0,74 \\
\hline CF_FPUX100P_PROM & 0,000 & 0,147 & 0,003 & 0,301 & 0,046 & 0,084 & 0,16 \\
\hline CF_JCIERVA_PROM & 0,004* & $0,801 *$ & $-0,002$ & 0,139 & 0,068 & 0,079 & 0,48 \\
\hline CF_JCIERVAx100P_PROM & $7,933 \mathrm{E}-6$ & 0,013 & 0,000 & $-0,07$ & 0,023 & 0,224 & 0,06 \\
\hline CF_RyCAJAL_PROM & 0,004* & $0,700 *$ & 0,001 & 0,052 & 0,077 & 0,093 & 0,51 \\
\hline CF_RyCAJALx100P_PROM & $-2,608 \mathrm{E}-5$ & $-0,051$ & $9,777 \mathrm{E}-5$ & 0,055 & 0,023 & 0,267 & 0,07 \\
\hline CF_TESIS_PROM & $0,149 *$ & $0,838^{*}$ & 0,061 & 0,101 & 1,999 & 0,0688 & 0,79 \\
\hline CF_TESISx100P_PROM & 0,002 & 0,268 & 0,002 & 0,118 & 0,259 & 0,254 & 0,14 \\
\hline
\end{tabular}

*Nivel de significación 0,01 .

**Nivel de significación 0,05. 


\section{CONCLUSIONES}

En este trabajo se analiza la relación entre la antigüedad, el tamaño y la ratio estudiantes-profesor de las universidades públicas españolas con los indicadores de la actividad investigadora, de lo que se extrae las siguientes conclusiones.

1. Al dividir el conjunto de universidades españolas en dos grupos, utilizando la mediana, las de mayor y las de menor antigüedad, resulta que las universidades antiguas presentan mejores indicadores que las recientes en valores absolutos, durante la primera década del siglo XXI, de actividad investigadora, de innovación, de competitividad y de capacidad formativa. Pero cuando la comparación se establece relativizando tales indicadores por el tamaño (como el número de profesores), el resultado es muy diferente y solamente se constatan diferencias significativas en el número de becas FPU y en el número de tesis defendidas), pero no hay diferencias significativas para los indicadores de actividad científica, innovación y competitividad.

Respecto a la hipótesis $\mathrm{H} 1$, hay que concluir que las universidades más antiguas presentan indicadores mayores que las más recientes en producción científica, en innovación, en competitividad y en capacidad formativa para indicadores absolutos, pero esto no se cumple para indicadores relativizados por número de profesores. En este caso solamente para el número de becas FPU y de tesis defendidas, las universidades antiguas presentan indicadores claramente mayores.

No obstante, hay que tener presente la elevada correlación entre la antigüedad y el tamaño. Por esto, descontando el efecto tamaño de la universidad medido por el número de profesores (también ocurre lo mismo si se utiliza el número de estudiantes), se comprueba que la antigüedad de la universidad no produce tantas diferencias significativas en los indicadores de la actividad investigadora.

2. El análisis de regresión aporta información adicional. En términos generales, la antigüedad de la universidad siempre que influye en los indicadores de la actividad científica, lo hace junto al tamaño de la universidad (número de profesores) pero con menor significación (normalmente al $5 \%$ frente al $1 \%$ del tamaño) y con menor fuerza (véase los coeficientes Beta que son menores que en el caso del tamaño). Así, la antigüedad influye de manera significativa, junto al tamaño, al explicar el número de becas FPU, número de proyectos del Programa Marco, y diversos indicadores de innovación (número de empresas spin-off, ingresos debidos a contratos de I+D y consultorías y a prestación de servicios, tanto en valor absoluto como por número de profesores, y los ingresos por patentes). Pero, con la excepción del número de becas FPU, en todos esos casos lo hace con signo negativo. Los años le pesan a las universidades en esos indicadores de competitividad (proyectos del Programa Marco) y de innovación.

3. Si la división de las universidades se realiza por el número de profesores, recurriendo a la mediana, el resultado es que las universidades con mayor número de profesores presentan mayores indicadores de la actividad investigadora en valores absolutos, como era de esperar, aunque con diferente magnitud, porque la diferencia es mayor en varios de los indicadores de innovación, en becas FPU o número de tesis. Además, se presentan diferencias cuasi significativas para indicadores relativos al número de profesores para ingresos por prestación de servicios y el número de becas FPU. Por tanto, en términos generales respecto a $\mathrm{H} 2$ hay que concluir que las universidades mayores presentan mayores indicadores de actividad investigadora en valores absolutos, pero no así cuando esos indicadores se relativizan, y además se identifican en qué indicadores su efecto es mayor, puesto que hay magnitudes muy diferentes.

4. Considerando en un modelo conjuntamente, el número de profesores, la antigüedad y la ratio estudiantes por profesor, el número de profesores es la variable determinante en casi todos los casos para explicar los indicadores de la actividad investigadora en los que se produce un ajuste aceptable. $Y$ esto ocurre para la casi totalidad de indicadores en valor absoluto y con signo positivo.

5. Respecto al análisis de las diferencias entre universidades por el número de estudiantes (ya sea número total o número de estudiantes equivalentes a tiempo completo), las conclusiones son básicamente las mismas que por el número de profesores. Como algo testimonial, es de destacar que para el caso del número de becas FPU por cada 100 profesores, el grupo de universidades con más estudiantes tiene un número mayor de estas becas.

6. En cuanto a la comparación entre universidades con mayor y menor ratio de estudiantes por profesor, no hay diferencias significativas salvo para los ingresos por licencias (a favor de las universidades con menor ratio) y también hay diferencias cuasi significativas para los ingresos por licencias por profesor, para el número de proyecto del Programa Marco por cada 100 profesores y para el número medio de becas FPI y FPU. La hipótesis H3 hay que rechazarla en términos generales, con las excepciones señaladas, de manera que la ratio estudiantes profesor no incide en los indicadores de actividad científica. 
7. Al considerar el modelo conjunto con número de profesores y antigüedad de la universidad, la ratio estudiantes por profesor es el motivo exclusivo de diferencias significativas en el número de sexenios por cada 100 profesores, en el número de proyectos conseguidos del Plan Nacional por cada 100 profesores y en el número de documentos no citados. Como ya se ha comentado, eso es debido a un grupo de universidades que son eficaces en la consecución de proyectos y de sexenios a pesar de estar entre las de mayor ratio de estudiantes por profesor.

En definitiva, estos datos confirman, el menos para el período analizado, que en España las universidades más antiguas tienen mejores indicadores absolutos, pero no relativos (según número de profesores), de actividad investigadora y que tal efecto tiene mucho que ver con el tamaño de la universidad. No obstante, llama la atención la relación negativa entre antigüedad y determinados indicadores referidos a innovación. La orientación a la innovación, medida con estos indicadores, suele ser menor a más antigüedad. La antigüedad es acompañada por la influencia del tamaño. Es decir, de acuerdo con lo comentado, siempre que la antigüedad influye en un indicador de la actividad investigadora lo hace junto a la influencia del tamaño pero con menor fuerza. Puesto que la heterogeneidad entre universidades referida a la ratio estudiantes-profesor es menor, tampoco es determinante la influencia en los indicadores de la actividad investigadora de este ratio, salvo para las excepciones mencionadas (como el número de sexenios y proyectos del Plan Nacional ambos relativizados por el número de profesores). Es el tamaño, medido por el número de profesores o de estudiantes, lo que marca la diferencia para la actividad científica, la innovación, la competitividad y la capacidad formativa, pero en valores absolutos que no en los relativos y con diferente intensidad según que indicador.

Por último, es necesario tener en consideración que se ha trabajado con los datos medios de la década, que no recogen la evolución experimentada por los indicadores a lo largo de esos años. Además, no debe olvidarse la existencia de universidades con perfiles diferentes, unas más técnicas que otras, o estructuras de titulaciones distintas que hacen a unas más propensas que otras a la innovación o a la producción científica, sin olvidar la dificultad que conlleva su medida según las áreas científicas.

\section{BIBLIOGRAFÍA}

Abramo, G.; Andrea, C.; Solazzi, M. (2012). A bibliometric tool to assess the regional dimension of university-industry research collaborations. Scientometrics, vol. 91 (1), 955-975.

Acs, Z. J.; Anselin, L.; Varga, A. (2002). Patents and innovation counts as measures of regional production of new knowledge. Research Policy, vol. 31, 1069-1085.
Barnett, R., ed. (2012): The future University. Ideas and Possibilites. International Studies in Higher Education. New York: Routledge, Taylor \& Francis Group.

Bordons, M.; Sancho, R.; Morillo, F.; Gómez, I. (2010). Perfil de actividad científica de las universidades españolas en cuatro áreas temáticas: un enfoque multifactorial. Revista Española de Documentación Científica, vol. 33 (1), 9-33.

Chen, K.; Liao, P. (2012). A comparative study on world university rankings: a bibliometric survey. Scientometrics, vol. 92 (1), 89-103.

Docampo, D. (2012). Adjusted sum of institutional scores as an indicator of the presence of university systems in the ARWU ranking. Scientometrics, vol. 90 (1), 701-713.

Docampo, D. (2011). On using the Shanghai ranking to assess the research performance of university systems. Scientometrics, vol. 86 (1), 77-92.

Docampo, D.; Herrera, F.; Luque-Martínez, T.; TorresSalinas, D. (2012). Efecto de la agregación de universidades españolas en el ranking de Shanghai (ARWU): caso de las comunidades autónomas y los campus de excelencia". El profesional de la información, vol. 21 (4), 428-432.

Etzkowitz, H.; Leydesdorff, L. (1998). The endless transition: A "triple helix" of university-industrygovernment relations. Minerva, vol. 36, 203-208.

Filippo, D.; Sanz-Casado, E.; Urbano Salido, C.; Ardanuy, J.; Gómez-Caridad, J. (2011). El papel de las bases de datos institucionales en el análisis de la actividad científica de las universidades. Revista Española de Documentación Científica, vol. 34 (2), 165-189.

García, J.A.; Rodríguez-Sánchez, R.; Fdez-Valdivia, J. ; Robinson-García, N.; Torres-Salinas, D. (2012). Mapping Academic Institutions According to Their Journal Publication Profile: Spanish Universities as a Case Study. Journal of the American Society for Information Science and Technology, vol. 63 (11), 2328-2340.

Hernández Armenteros, J.; Pérez García, J.; Hernández Chica, J. (2010). La universidad española en cifras. Madrid: Conferencia de Rectores de las Universidades Españolas.

Hernández Armenteros, J. (2008). La universidad española en cifras. Madrid: Conferencia de Rectores de las Universidades Españolas.

Hernández Armenteros, J. (2006). La universidad española en cifras. Madrid: Conferencia de Rectores de las Universidades Españolas.

Hernández Armenteros, J. (2004). La universidad española en cifras. Madrid: Conferencia de Rectores de las Universidades Españolas.

Hernández Armenteros, J. (2002). La universidad española en cifras. Madrid: Conferencia de Rectores de las Universidades Españolas.

Herranz, N.; Ruiz-Castillo, J. (2012). The end of the "European Paradox". Scientometrics. DOI 10.1007/ s11192-012-0865-8, [19-10-2012].

IBM SPSS Advanced Statistics (2011). ftp://public. dhe.ibm.com/software/analytics/spss/documenta- 
tion/statistics/20.0/es/client/Manuals/IBM_SPSS_ Advanced_Statistics.pdf [DOL 4-XI-2012].

Jaffe, A. B. (1989). Real effects of academic research. American Economic Review, vol. 79 (5), 957-970.

Jiménez-Contreras, E.; Robinson-García, N. ; CabezasClavijo, A. (2011). Productividad e impacto de los investigadores españoles: umbrales de referencia por áreas científicas. Revista Española de Documentación Científica, vol. 34 (4), 505-526.

Li, F.; Yi, Y.; Guo, X.; Qi, W. (2012). Performance evaluation of research universities in Mainland China, Hong Kong and Taiwan: based on a two-dimensional approach. Scientometrics, vol. 90 (2), 531-542.

Lin, X.; Roberts, J. (2012): A stages approach to the internationalization of higher education? The entry of UK universities into China. The Service Industries Journal, vol. 32 (7), 1011-1038.

Luque Martínez, T. (2012). Técnicas de análisis de datos en investigación de mercados. Madrid: Pirámide.

OECD, (2007). OECD Science, Technology and Industry Scoreboard 2007. http://www.oecd.org/science/inno/oecdsciencetechnologyandindustryscoreboard2007.htm, [19-10-2012]

OECD (2011). OCDE Science, Technology and Industry Scoreboard 2011: Innovation and Growth in Knowledge Economies http://www.oecd.org/ innovation/innovationinsciencetechnologyandindustry/oecdsciencetechnologyandindustryscoreboard2011innovationandgrowthinknowledgeeconomies.htm, [19-10-2012].

Rothblat, S. (2012). The future isn't waiting. En Barnett, R. (ed.), The future University. Ideas and Possibilites. International Studies in Higher Education. New York: Routledge, Taylor \& Francis Group.

Sanz-Casado. E. (2012). Lanzamiento del Observatorio IUNE, una herramienta para el seguimiento de la actividad científica de las universidades españolas. Revista Española de Documentación Científica, vol. 35 (3), 503-505.

Sun, Y.; Liu, F. (2012). Measuring international traderelated technology spillover: a composite approach of network analysis and information theory. Scientometrics, vol. 94 (3), 963-979.

Torres-Salinas, D.; Jiménez-Contreras, E. (2012). Hacia las unidades de bibliometría en las universidades: modelo y funciones. Revista Española de Documentación Científica, vol. 35 (3), 469-480.

Urdín, C.; Morillo, F. (2000). Producción científica de Andalucía en las bases de datos Science Citation Index e Índice Español de Ciencia y Tecnología. Revista Española de Documentación Científica, vol. 23 (4), 379-394. 
ANEXO I

RELACIÓN DE UNIVERSIDADES PÚBLICAS PRESENCIALES ESPAÑOLAS

\begin{tabular}{|c|c|}
\hline & UNIVERSIDAD \\
\hline EHU & País Vasco \\
\hline UA & Alicante \\
\hline UAB & Autónoma de Barcelona \\
\hline UAH & Alcalá de Henares \\
\hline UAL & Almería \\
\hline UAM & Autónoma de Madrid \\
\hline UB & Barcelona \\
\hline UBU & Burgos \\
\hline UC3M & Carlos III \\
\hline UCA & Cádiz \\
\hline UCLM & Castilla-La Mancha \\
\hline UCM & Complutense de Madrid \\
\hline UCO & Córdoba \\
\hline UDC & Coruña, A \\
\hline UDG & Girona \\
\hline UDL & Lleida \\
\hline UGR & Granada \\
\hline UHU & Huelva \\
\hline UIB & Illes Balears \\
\hline UJAEN & Jaén \\
\hline UJI & Jaume I de Castellón \\
\hline ULL & La Laguna \\
\hline ULPGC & Palmas (Las) \\
\hline UM & Murcia \\
\hline
\end{tabular}

\begin{tabular}{|c|c|}
\hline & UNIVERSIDAD \\
\hline UMA & Málaga \\
\hline UMH & Miguel Hernández de Elche \\
\hline UNAVARRA & Pública de Navarra \\
\hline UNEX & Extremadura \\
\hline UNICAN & Cantabria \\
\hline UNILEON & León \\
\hline UNIOVI & Oviedo \\
\hline UNIRIOJA & La Rioja \\
\hline UNIZAR & Zaragoza \\
\hline UPC & Politécnica de Cataluña \\
\hline UPCT & Politécnica de Cartagena \\
\hline UPF & Pompeu Fabra \\
\hline UPM & Politécnica de Madrid \\
\hline UPO & Pablo de Olavide \\
\hline UPV & Politécnica de Valencia \\
\hline URJC & Rey Juan Carlos \\
\hline URV & Rovira i Virgili \\
\hline US & Sevilla \\
\hline USAL & Salamanca \\
\hline USC & Santiago \\
\hline UV & Valencia (Est. General) \\
\hline UVA & Valladolid \\
\hline UVIGO & Vigo \\
\hline
\end{tabular}

